hep-th/9604070

MRI-PHY/96-12

\title{
Duality and Orbifolds
}

\author{
Ashoke Sen円2 \\ Mehta Research Institute of Mathematics \\ and Mathematical Physics \\ 10 Kasturba Gandhi Marg, Allahabad 211002, INDIA
}

\begin{abstract}
We construct several examples where duality transformation commutes with the orbifolding procedure even when the orbifolding group does not act freely, and there are massless states from the twisted sector at a generic point in the moduli space. Often the matching of spectrum in the dual theories is a result of non-trivial identities satisfied by the coefficients of one loop tadpoles in the heterotic, type II and type I string theories.
\end{abstract}

\footnotetext{
${ }^{1}$ On leave of absence from Tata Institute of Fundamental Research, Homi Bhabha Road, Bombay 400005, INDIA

${ }^{2}$ E-mail: sen@mri.ernet.in, sen@theory.tifr.res.in
} 


\section{Introduction}

Over the last few years many examples of pairs of string theories have been constructed such that they are dual to each other in the non-perturbative sense. In establishing the duality between a pair of theories, one often uses the power of supersymmetry and the associated non-renormalization theorems to compare physical quantities which can be calculated in both the theories. Once duality is established to a resonable level of confidence, one can then use it to compute physical quantities in one theory by mapping it to a simpler problem in the dual theory. This procedure has yielded many non-perturbative results in string theories which were beyond the scope of the conventional perturbative formulation of string theory.

However, in order to be able to make full use of the power of duality, one should have an a priori means of determining when two theories might be dual to each other. This is particularly relevant in case of pairs of theories with little or no supersymmetry, where the usual non-renormalization theorems are absent, and hence we do not have the tools for testing the duality conjecture. Some progress has been made in this direction, and many of the duality conjectures have been 'derived' from other duality conjectures by a set of well defined operations. This involves construction of new pairs of dual theories by taking orbifolds of known pairs or a slight variant of this idea 11, 2]. More specifically, suppose theory $A$ compactified on a manifold $K_{A}$ is known to be dual to the theory $B$ compactified on the manifold $K_{B}$. Let us now compactify both theories further on a manifold $M$. Let $G_{A}$ be a group of discrete symmetries acting on the first theory, and $G_{B}$ be the image of this group in the second theory. Then naively one would expect that the first theory modded out by the group $G_{A}$ will be dual to the second theory modded out by the group $G_{B}$. This procedure yields correctly a pair of dual theories when the duality transformation relating the two theories is part of the $T$-duality group, but it does not always work when the duality is part of the more general $U$-duality group [2]. Still, it has quite often lead correctly to a pair of dual theories. The purpose of this paper is to investigate this procedure in some detail through some examples.

For simplicity of argument let us restrict our discussion to the case where $G_{A}\left(G_{B}\right)$ is a $Z_{2}$ group generated by the element $g_{A}\left(g_{B}\right)$. Then $G_{A}=\left\{1, g_{A}\right\}$ and $G_{B}=\left\{1, g_{B}\right\}$. By an abuse of notation we shall denote the group $G_{A}\left(G_{B}\right)$ by its generator $g_{A}\left(g_{B}\right)$, and denote the theory $A(B)$ on $M \times K_{A}\left(M \times K_{B}\right)$ modded out by $G_{A}\left(G_{B}\right)$ as theory 
$A$ on $\left(M \times K_{A}\right) / g_{A}$ (theory $B$ on $\left.\left(M \times K_{B}\right) / g_{B}\right)$. Let $h_{A}\left(h_{B}\right)$ denote the part of the $Z_{2}$ transformation representing geometric action on the internal manifold $K_{A}\left(K_{B}\right)$ as well as any internal symmetry transformation and $s$ be the part of the transformation representing geometric action on the manifold $M$. Then $g_{A}=s \cdot h_{A}$ and $g_{B}=s \cdot h_{B}$. We shall now consider three separate cases:

1. Suppose $s$ acts freely on $M$. In this case the manifold $\left(M \times K_{A}\right) / s \cdot h_{A}$ can be regarded as a fiber bundle with base space $M / s$ and fiber $K_{A}$, and similarly the manifold $\left(M \times K_{B}\right) / s \cdot h_{B}$ can be regarded as a fiber bundle with base space $M / s$ and fiber $K_{B} . h_{A}\left(h_{B}\right)$ represents twist on the fiber as we move along the order two closed cycles of $M / s$ connecting the points $P$ and $s(P)$ on $M$. The duality transformation between the two theories can be regarded as a duality between the fibers at every point in $M / s$. We expect this duality to be valid when the volume of $M$ is large, since locally $M / s$ is nearly flat and hence the required duality reduces to the original duality conjecture between theory $A$ on $K_{A}$ and theory $B$ on $K_{B}$. We can then adibatically reduce the volume of $M$ to any desired size without destroying the duality in this process. This argument, due to Vafa and Witten[2] has been named adiabatic argument.

2. Next we consider the case where $s$ does not act freely on $M$, but it does not leave the whole of $M$ invariant. In other words $s$ has non-trivial action on $M$ and has isolated fixed points (or fixed hyper-planes). In this case the manifold $\left(M \times K_{A}\right) / s \cdot h_{A}$ can still be regarded as a fiber bundle with base space $M / s$ and fiber $K_{A}$ but the description breaks down at the orbifold points of $M / s$. At these points the fiber is $K_{A} / h_{A}$. A similar description holds for the other theory. Thus we can again try to establish the duality between the two theories by taking $M$ to be of large volume and applying the duality fiberwise. But this breaks down at the orbifold points and hence the argument is not as strong as in the previous case. Nevertheless, this procedure (or a variation of the procedure) has yielded many correct pairs of dual theories. Let us now subdivide these models into two classes:

(a) Suppose that even though $s$ does not act freely on $M, s \cdot h_{A}\left(s \cdot h_{B}\right)$ acts freely on $M \times K_{A}\left(M \times K_{B}\right)$. In other words $h_{A}\left(h_{B}\right)$ acts freely on $K_{A}\left(K_{B}\right)$ and hence the fibers at the orbifold points of $M / s$ are non-singular. Among other things, this implies that at a generic point in the moduli space (say where 
$M \times K_{A}$ and $M \times K_{B}$ have large volume) there are no massless states from the twisted sector. Thus the spectrum of massless states in the two theories after orbifolding match trivially, since they come from the untwisted sector. There could be special points in the moduli space where twisted sector states become massless, and comparing the effects of these massless states in the two theories provide a non-trivial check on the duality conjecture. The first such construction was given in ref.[四]. Most of the examples of dual pairs, constructed by the orbifolding procedure, fall either in this class, or in the previous class where the adiabatic argument is applicable.

(b) We can also consider the case where $h_{A}\left(h_{B}\right)$ does not act freely on $K_{A}\left(K_{B}\right)$. In this case $s \cdot h_{A}\left(s \cdot h_{B}\right)$ does not act freely on $M \times K_{A}\left(M \times K_{B}\right)$, and often we have massless states from the twisted sector at a generic point in the moduli space. Thus a non-trivial test of the duality between the two resulting theories is provided by comparing the spectrum of massless states from the twisted sectors in the two theories. Most of our analysis in this paper will focus on this class of theories. We shall construct several examples of dual pairs where on each side there are massless states from the 'twisted sector', and verify that the spectrum of these massless states in the two theories agree.

3. Finally consider the case where $s$ acts trivially on $M$, i.e. it leaves the whole of $M$ invariant. In this case the manifold $M \times K_{A} / s \cdot h_{A}$ does not have the structure of a fiber bundle with fiber $K_{A}$ even locally. In fact the fiber is everywhere $K_{A} / h_{A}$. Thus we cannot hope to establish the duality between the two resulting theories by applying the original duality on the fibers. Thus the case for equivalence between the two resulting theories is weakest in this case, and indeed, as we shall discuss, in most examples of this kind the duality does not hold.

In the next section we shall consider several examples of dual pairs of the kind 2(b) and show that the spectrum of massless states from the twisted sector matches in the two theories constructed this way. Already examples of dual pairs of this kind were constructed in ref.[3] (see also [4]) where one side of the theory involved $M$-theory orbifolds and were shown to reproduce the conjectures of refs. [5, 6]. In this paper we shall focus on examples where both sides involve orbifolds of string theories, so that one can independently compute the spectrum of 'twisted sector states' in both theories and make a 
meaningful comparison. As we shall see, in several examples, part of the massless spectrum arises from the background elementary strings which need to be introduced in the theory to cancel one loop tadpoles[7], and only after taking into account the fields living on these elementary strings, the massless spectrum in the two theories agree.

\section{Examples of Dual Pairs}

In this section we shall construct examples of dual pairs of theories using the procedure outlined in the introduction. First we shall introduce some notations for various symmetry transformations that we shall be using in our analysis. In type IIA or IIB string theories, we shall denote by $F_{L}$ the space-time fermion number arising in the left-moving sector on the world sheet. Both these theories are invariant under the $Z_{2}$ group of transformations generated by $(-1)^{F_{L}}$, whose effect on the bosonic fields in the theory is to change the sign of all the fields arising in the Ramond-Ramond (RR) sector, leaving the fields from the Neveu-Schwarz Neveu-Schwarz (NS) sector invariant. Type IIB theory is also invariant under the world-sheet parity transformation which we shall denote by $\Omega$. Acting on the massless bosonic sector states in the theory, it changes the sign of the anti-symmetric tensor field in the NS sector, and the scalar and the rank four anti-symmetric tensor field in the RR sector, leaving the other fields invariant. Any string theory compactified on a $2 n$ dimensional torus $T^{2 n}$ is invariant under a change of sign of all the $2 n$ coordinates on the torus. We shall denote this transformation by $\mathcal{I}_{2 n}$. For heterotic string theory compactified on $T^{2 n}$, we shall also define the transformation $\mathcal{I}_{2 n+16,2 n}$ to be the one that changes the sign of all the coordinates of the signature $(2 n+16,2 n)$ Narain lattice.

The non-perturbative duality symmetries that we shall be using for constructing dual pairs of theories are the following. First of all, in ten dimensions, type IIB theory has an $\mathrm{SL}(2, \mathrm{Z}) S$-duality symmetry [8]. We shall denote by $S$ the non-trivial $Z_{2}$ transformation that takes the string coupling to its inverse for vanishing axion field. For type IIB or IIA theory compactified on $T^{4}$, the full $U$-duality group $S O(5,5 ; Z)$ contains a $Z_{2}$ transformation that changes the sign of the dilaton field, and takes the field strength associated with the rank two antisymmetric tensor field in the NS sector to its dual[9]. We shall denote this by $\sigma$. Finally we shall denote by $\eta$ the string-string duality transformation that relates the type IIA string theory compactified on $K 3$ to heterotic string theory compactified on $T^{4}[8]$. 
Before we discuss construction of dual pairs of type 2(b) mentioned in the introduction, let us discuss some examples of type 3 which do not work.

- Consider first type IIB theory in ten dimensions. In this case it can be easily seen that conjugation by $S$ takes $(-1)^{F_{L}}$ to $\Omega$. Now, type IIB theory modded out by $(-1)^{F_{L}}$ gives type IIA theory, whereas the same theory modded out by $\Omega$ gives the type I string theory with gauge group $\mathrm{SO}(32)$. These two theories are clearly not the same.

- For type IIA theory on $T^{4}$, the transformation $\sigma$ converts $(-1)^{F_{L}}$ to $\mathcal{I}_{4}[$ g]. Type IIA on $T^{4}$ modded out by $(-1)^{F_{L}}$ gives type IIB on $T^{4}$, whereas the same theory modded out by $\mathcal{I}_{4}$ gives type IIA on $K 3$ orbifold. Again the two theories are clearly not equivalent.

- Finally let us consider the string-string duality transformation $\eta$. This maps the symmetry $(-1)^{F_{L}}$ on the type IIA side to $\mathcal{I}_{20,4}$ on the heterotic side[2]. Modding out by $(-1)^{F_{L}}$ gives type IIB on $K 3$ on the type II side; whereas modding out by $\mathcal{I}_{20,4}$ gives an inconsistent theory on the heterotic side because of problem with left-right level matching[2].

Note that in each of the cases discussed above we did not compactify the original model further on another manifold $M$ and combined the original $Z_{2}$ symmetry with a non-trivial action on this manifold. Thus these examples all belong to class 3 where the argument for duality is the weakest. We shall now show that in each of the above cases, when we combine the transformations discussed above with a $Z_{2}$ action on the rest of the manifold we can get sensible dual pairs. The case where the $Z_{2}$ action on the rest of the manifold is free has already been discussed elsewhere 2, 9], so we shall focus our attention to the cases where the $Z_{2}$ action on the manifold $M$ is not free.

\subsection{Type IIB on $T^{4} /(-1)^{F_{L}} \cdot \mathcal{I}_{4}$ and type IIB on $T^{4} / \Omega \cdot \mathcal{I}_{4}$}

The ten dimensional $S$-duality transformation $S$ relates the symmetries $(-1)^{F_{L}}$ and $\Omega$ in the type IIB theory. We compactify the theory on $T^{4}$ and combine this internal symmetry with the reflection $\mathcal{I}_{4}$ of $T^{4}$. This leads to the dual pair type IIB on $T^{4} /(-1)^{F_{L}} \cdot \mathcal{I}_{4}$ and type IIB on $T^{4} / \Omega \cdot \mathcal{I}_{4}$. An $R \rightarrow(1 / R)$ duality transformation on one of the circles of $T^{4}$ converts the type IIB theory to type IIA theory, and the transformation $(-1)^{F_{L}} \cdot \mathcal{I}_{4}$ to 
$\mathcal{I}_{4}$. Thus the first theory is related by a $T$-duality transformation to type IIA on $T^{4} / \mathcal{I}_{4}$, which is just the type IIA theory compactified on a $K 3$ orbifold. On the other hand, making a $T$-duality transformation on all the four circles of $T^{4}$, the symmetry $\Omega \cdot \mathcal{I}_{4}$ can be mapped to $\Omega[10]$. Thus the second theory is related by a $T$-duality transformation to type IIB on $T^{4} / \Omega$, which is just the type I theory on $T^{4}$. Thus the dual pair constructed here is related by $T$-duality to the well known dual pair, type IIA on $K 3$ and type I on $T^{4}$ (which in turn is known to be equivalent to heterotic string theory on $T^{4}$ [11).

Using this prescription one can in fact derive a precise map between the moduli spaces of the two theories. The particularly interesting aspect of this is the map between the moduli coming from the 'twisted sector' states. For this it is best to work with the original dual pair instead of their $T$-dual versions. For the type IIB on $T^{4} /(-1)^{F_{L}} \cdot \mathcal{I}_{4}$, it has been argued by Kutasov [12] (see also [13, 14]) that the twisted sector states live on the sixteen NS five-branes of the type IIB theory, moving on $T^{4} / \mathcal{I}_{4}$. Each such five-brane supports one vector multiplet of the non-chiral $N=2$ supersymmetry algebra in six dimensions. The vector-multiplet moduli associated with the blowing up modes in the type IIA description correspond in the type IIB description to the locations of these five-branes on $T^{4} / \mathcal{I}_{4}$. On the other hand, for type IIB on $T^{4} / \Omega \cdot \mathcal{I}_{4}$, the 'twisted sector' states live on sixteen RR five-branes moving on $T^{4} / \mathcal{I}_{4}$ [10]. Again each of these five-branes support one vector multiplet of the $N=2$ supersymmetry algebra, and the vector multiplet moduli from the 'twisted sector' correspond to the locations of the RR five-branes on $T^{4} / \mathcal{I}_{4}$. The $S$-duality transformation in the ten dimensional type IIB theory precisely transforms an NS fivebrane to an RR five-brane and vice versa. This gives us a precise map between the moduli fields in the two theories: the moduli coming from the untwisted sectors get mapped into each other by the usual rules of $S$-duality transformation in the ten dimensional type IIB theory, and the locations of the NS five-branes in one theory get mapped to the locations of the RR five-branes in the dual theory. This story, already anticipated in refs. [12, 13, 15] in this case, will repeat itself in every example that we shall consider below.

\subsection{Type IIB on $T^{8} /(-1)^{F_{L}} \cdot \mathcal{I}_{8}$ and type IIB on $T^{8} / \Omega \cdot \mathcal{I}_{8}$}

In order to construct this dual pair, we compactify type IIB theory on an eight dimensional torus $T^{8}$ and repeat the procedure of the last subsection with $\mathcal{I}_{4}$ replaced by $\mathcal{I}_{8}$. By an $R \rightarrow(1 / R)$ duality transformation in one of the circles of $T^{8}$, the first theory is mapped to type IIA on $T^{8} / \mathcal{I}_{8}$. On the other hand by making $R \rightarrow(1 / R)$ duality transformation 
on all the circles, the second theory is mapped to type $I$ theory on $T^{8}$. At a generic point in the moduli space, the 'twisted sector states' in the second theory correspond to sixteen vector multiplets of the non-chiral $N=16((8,8))$ supersymmetry algebra in two dimensions. Viewed as the orientifold IIB on $T^{8} / \Omega \cdot \mathcal{I}_{8}$, the 'twisted sector' states live on the sixteen $\mathrm{RR}$ one-branes moving on $T^{8} / \mathcal{I}_{8}$, and the $16 \times 8$ scalars from the sixteen vector multiplets may be regarded as the locations of these sixteen one-branes on $T^{8} / \mathcal{I}_{8}$.

Let us now turn to the twisted sector states in the first theory by regarding it as type IIA on $T^{8} / \mathcal{I}_{8}$. First of all it is easy to see that there are no massless states from the sectors where either the left or the right moving fermions are in the NS sector, since this gives the total vacuum energy in the corresponding sector to be $(1 / 2)$. Thus the only possible massless states could come from the RR sector. To determine this spectrum let us work in the light cone gauge NSR formalism.f In the twisted RR sector, all the world-sheet fermions and bosons are anti-periodic, and hence, before the GSO projection, there is a unique $\mathcal{I}_{8}$ invariant ground state with zero energy associated with each of the 256 fixed points. The transformation $\mathcal{I}_{8}$, acting on the $\mathrm{RR}$ ground state, has the same effect as $(-1)^{f_{L}}(-1)^{f_{R}}$, where $f_{L}$ and $f_{R}$ are the world sheet fermion number operators on the left and the right moving sectors respectively. We shall work in the convention where GSO projection requires a Ramond sector state in the left to have $(-1)^{f_{L}}$ eigenvalue -1 and a Ramond sector state on the right to have $(-1)^{f_{R}}$ eigenvalue +1 , reflecting the choice of opposite space-time chiralities in the left and the right sector in the type IIA theory. This shows that all the $\mathcal{I}_{8}$ invariant $\mathrm{RR}$ ground states are odd under either the left or the right moving GSO operator and hence are projected out. P Thus there are no physical massless states from the twisted sector. (For definiteness we shall take the twisted sector RR states to be even under both $(-1)^{f_{L}}$ and $(-1)^{f_{R}}$. Thus they survive the GSO projection on the right, but fails to survive the GSO projection on the left.)

This seems to lead to a contradiction, since duality implies that this theory must have sixteen vector multiplets from the twisted sector. There is however a subtle effect which

\footnotetext{
${ }^{3} \mathrm{By}$ an abuse of notation we shall refer to the supermultiplet, obtained by dimensional reduction of the vector multiplet of the $\mathrm{N}=4$ supersymmetry algebra in four dimensions, as the vector multiplet of the two dimensional supersymmetry algebra.

${ }^{4}$ Since light cone gauge analysis gives us the spectrum for $k^{+} \neq 0$ states only, working in this gauge can sometimes be deceptive for chiral theories in two dimensions. Since the theory we are analysing at present is non-chiral, working in the light cone gauge does not cause any problem.

${ }^{5}$ When viewed as compactification of type IIB theory on $T^{8} /(-1)^{F_{L}} \cdot \mathcal{I}_{8}$, GSO projection will require both $(-1)^{f_{L}}$ and $(-1)^{f_{R}}$ eigenvalues to be +1 . But acting on RR ground states $(-1)^{F_{L}} \cdot \mathcal{I}_{8}$ will have the effect of $-(-1)^{f_{L}}(-1)^{f_{R}}$. Thus $(-1)^{F_{L}} \cdot \mathcal{I}_{8}$ invariant states still fail to survive the GSO projection.
} 
restores these vector multiplets in this theory. As was shown by Vafa and Witten[[], some string theories in two dimensions can have tadpoles of the NS sector anti-symmetric tensor field of the form:

$$
2 \pi i n B
$$

where

$$
B=\frac{1}{2} \epsilon^{\mu \nu} B_{\mu \nu}
$$

is normalized so as to have periodicity one, and $n$ is a constant. The theory is inconsistent in the presence of such a tadpole, but this can be overcome by placing $n$ elementary strings moving in the internal manifold, whose word-volume fills up the physical space-time. Each of these strings would give a vector multiplet of the $N=16$ supersymmetry algebra in two dimensions, with its eight scalar components labelling the location of the string in the internal space. Thus if in the present case $|n|$ turns out to be 16 , then we would get complete agreement between the spectrum of massless states in the two theories. This would also give a map between the moduli spaces in the two theories as in the previous case. $]^{0}$ To see this we work with the original pair, type IIB on $T^{8} /(-1)^{F_{L}} \cdot \mathcal{I}_{8}$ and type IIB on $T^{8} / \Omega \cdot \mathcal{I}_{8}$. The moduli from the untwisted sector states in the two theories are mapped into each other by the usual $S$-duality transformation rules of the ten dimensional theory. The moduli of the twisted sector states in the first theory are represented by the locations of the sixteen elementary type IIB strings on $T^{8} / \mathcal{I}_{8}$ (note that under the $R \rightarrow 1 / R$ duality that takes us from the type IIB to the type IIA description, the elementary type IIB string goes over to the elementary type IIA string). On the other hand the moduli of the twisted sector states of the second theory are represented by the locations of the sixteen RR strings (D-strings) on the internal manifold $T^{8} / \mathcal{I}_{8}$. The $S$-duality transforms the elementary type IIB string to a D-string, and hence the locations of the elementary strings in the first theory get mapped to those of the $D$-strings in the second theory.

Thus all that remains to be shown is that the number $|n|$ of elementary strings, required to cancel the tadpole of the $B$ field, is indeed 16 in the present case. This number was calculated in ref. [7] and is given by (up to a sign):

$$
n=-\frac{1}{8 \pi} \int_{\partial \mathcal{M}} d \tau_{1}\left(\frac{1}{\tau_{2}}-\frac{4 i \partial_{\tau} \eta}{\eta}\right) A_{M}(q)
$$

\footnotetext{
${ }^{6}$ In a two dimensional theory the word moduli space is a misnomer, but we shall continue to use it anyway to denote the configuration space of massless scalars in the theory.
} 
where $\mathcal{M}$ denotes a fundamental region of the moduli space of a torus, $\tau=\tau_{1}+i \tau_{2}$ is the complex coordinate labelling this moduli space, $\eta$ is the Dedekind eta function, and $A_{M}(q)$ is the elliptic genus of the conformal field theory associated with the eight transverse coordinates, defined as the partition function of this conformal field theory in the (even,odd) spin structure where even and odd refer to the left and the right moving sectors on the world-sheet. Defining $q=e^{2 \pi i \tau}$, and noting that $\int_{\partial \mathcal{M}} d \tau_{1}=\oint(d q / 2 \pi i q)$ we see that the non-trivial contribution from the boundary $q=0$ to the above integral can be obtained by expanding the integrand in powers of $q$ and keeping the $q^{0}$ term in the expansion. In particular

$$
\frac{1}{\tau_{2}}-\frac{4 i \partial_{\tau} \eta}{\eta}=\frac{\pi}{3}-\frac{2 \pi}{\ln |q|}-8 \pi q+O\left(q^{2}\right)
$$

On the other hand $[7]$,

$$
A_{M}(q)=-\left(2 n_{N S, R}-n_{R, R}\right)+O(q),
$$

where $n_{N S, R}$ is the number of massless states in the NS-R sector that survives GSO projection from the left, weighted by $(-1)^{f_{R}}$ where $f_{R}$ denotes the world-sheet fermion number from the right. Note that in counting $n_{N S, R}$ we do not require the state to have survived GSO projection on the right. $n_{R, R}$ on the other hand is the number of massless states in the RR sector weighted by $(-1)^{f_{R}}$; in this case we do not require the state to have survived GSO projection either from the left or from the right. Using (2.4) and (2.5) in $(2.3)$ we get

$$
n=\frac{1}{24}\left(2 n_{N S, R}-n_{R, R}\right) .
$$

Thus it remains to compute $n_{N S, R}$ and $n_{R, R}$. First we shall compute $n_{R, R}$. This can get contribution from the untwisted sector as well as the twisted sector. Before GSO projection, and the projection by $\mathcal{I}_{8}$, there are $16 \times 16$ states from the untwisted sector, which transform under the tangent space $S O(8)$ group of the internal manifold as

$$
\left(8_{s}+8_{c}\right)_{L} \times\left(8_{s}+8_{c}\right)_{R}
$$

$8_{s}$ and $8_{c}$ denoting the two inequivalent spinor representations of $S O(8)$. $\mathcal{I}_{8}$ acts on these spinor representations of $S O(8)$ by changing the sign of $8_{s}$ but keeping $8_{c}$ invariant. Thus the states that survive the $\mathcal{I}_{8}$ projection are

$$
\left(8_{c}\right)_{L} \times\left(8_{c}\right)_{R}+\left(8_{s}\right)_{L} \times\left(8_{s}\right)_{R}
$$


Since we have adopted the convention that the action of $\mathcal{I}_{8}$ and $(-1)^{f_{R}}$ agree on the Ramond sector ground states on the right, $(-1)^{f_{R}}$ leaves $\left(8_{c}\right)_{R}$ invariant and changes the sign of $\left(8_{s}\right)_{R}$. Thus of the 128 states that survive the $\mathcal{I}_{8}$ projection, 64 are odd under $(-1)^{f_{R}}$ and 64 are even. As a result the contribution of these states to $n_{R, R}$, which counts the number of massless $\mathrm{RR}$ states weighted by $(-1)^{f_{R}}$, vanishes.

Let us now turn to the twisted sector. As mentioned before, there are 256 fixed points, and associated with each of them is a unique ground state of zero energy in the $\mathrm{RR}$ sector that is invariant under $\mathcal{I}_{8}$ (but does not survive the GSO projection on the left). According to the convention we have adopted, these all carry $(-1)^{f_{R}}=1$. This gives

$$
n_{R, R}=256 .
$$

Computation of $n_{N S, R}$ follows a similar pattern. The untwisted sector states in the massless sector before any projection transform under $\mathrm{SO}(8)$ as

$$
\left(8_{v}\right)_{L} \times\left(8_{s}+8_{c}\right)_{R},
$$

where $8_{v}$ denotes the vector representation of $\mathrm{SO}(8)$. This survives the GSO projection on the left. Under $\mathcal{I}_{8}, 8_{v}$ and $8_{s}$ changes sign, but $8_{c}$ remains invariant. Thus the state that survives $\mathcal{I}_{8}$ projection is $\left(8_{v}\right)_{L} \times\left(8_{s}\right)_{R}$. Since $\left(8_{s}\right)_{R}$ has $(-1)^{f_{R}}=-1$, these states contribute a net factor of -64 to $n_{N S, R}$. In the twisted sector, all the eight bosons on the left are antiperiodic and all the eight fermions on the left are periodic. This gives a total contribution of $(1 / 2)$ to the vacuum energy on the left, showing that there are no massless states from this sector. Thus we get

$$
n_{N S, R}=-64 .
$$

Using eqs.(2.6), (2.9) and (2.11) we finally get

$$
|n|=16,
$$

as required.

\subsection{Type IIA on $\left(T^{4} \times\left(T^{4}\right)^{\prime}\right) /(-1)^{F_{L}} \cdot \mathcal{I}_{4}^{\prime}$ and type IIA on $\left(T^{4} \times\right.$ $\left.\left(T^{4}\right)^{\prime}\right) / \mathcal{I}_{4} \cdot \mathcal{I}_{4}^{\prime}$}

Type IIA (or IIB) theory compactified on $T^{4}$ has a $Z_{2}$ self-duality transformation $\sigma$ described before. This converts the transformation $(-1)^{F_{L}}$ to $\mathcal{I}_{4}$. Let us compactify the 
theory further on a four torus (which we shall denote by $\left.\left(T^{4}\right)^{\prime}\right)$ and mod out the theory by combined operation of $(-1)^{F_{L}}$ (or $\mathcal{I}_{4}$ ) and the reflection $\mathcal{I}_{4}^{\prime}$ on this new torus. This leads to the dual pair of theories described in the title of this subsection.

We shall now compare the spectrum of twisted sector states in the two theories obtained this way. The first theory, after an $R \rightarrow 1 / R$ duality transformation in one of the circles of $\left(T^{4}\right)^{\prime}$ can be identified to type IIB on $\left(T^{4} \times\left(T^{4}\right)^{\prime}\right) / \mathcal{I}_{4}^{\prime}$, i.e. type IIB on $T^{4} \times K 3^{\prime}$ at the orbifold limit of $K 3^{\prime}$. The twisted sector states in this case come from the sixteen fixed points of $\left(T^{4}\right)^{\prime}$ under $\mathcal{I}_{4}$. Following Kutasov [12] it is more convenient to describe these in the original orbifold - type IIA on $\left(T^{4} \times\left(T^{4}\right)^{\prime}\right) /(-1)^{F_{L}} \cdot \mathcal{I}_{4}^{\prime}$ - as living on sixteen NS five-branes of the type IIA theory transverse to $\left(T^{4}\right)^{\prime} / \mathcal{I}_{4}^{\prime}$ and wrapped around $T^{4}$. In type IIA on $T^{4}$ such a configuration corresponds to the dual of the elementary string in six dimensions. Thus the twisted sector states can be regarded as living on the sixteen dual strings in six dimensions moving on $\left(T^{4}\right)^{\prime} / \mathcal{I}_{4}^{\prime}$.

In the second theory, the transformation $\mathcal{I}_{4} \times \mathcal{I}_{4}^{\prime}$ is simply the reflection $\mathcal{I}_{8}$ of the full eight dimensional torus. Thus this theory is just type IIA on $T^{8} / \mathcal{I}_{8}$, - the one we analysed in the previous subsection. As was found there, in this case there are no massless states from the conventional twisted sector, but cancellation of one loop tadpoles forces us to introduce sixteen elementary strings. There are extra massless states living on these sixteen elementary strings moving on $T^{8} / \mathcal{I}_{8}$. An elementary string in the ten dimensional type IIA string is also an elementary string in the six dimensional type IIA string obtained by compactifying the ten dimensional theory on $T^{4}$. Thus from the six dimensional view point, the 'twisted sector states' live on sixteen elementary strings moving on $\left(T^{4}\right)^{\prime} / \mathcal{I}_{4}^{\prime}$.

Comparison with the first theory shows that this is precisely what we would have expected to happen. Under the duality transformation $\sigma$ in the six dimensional theory, dual strings get converted to elementary strings and vice versa. This converts the sixteen dual strings in the first theory to sixteen elementary strings in the second theory. Besides showing that the spectrum in the two theories agree, this procedure again gives us the relation between the moduli fields in the two theories, in the untwisted as well as the twisted sector. 


\subsection{Type IIA on $\left(K 3 \times\left(T^{4}\right)^{\prime}\right) /(-1)^{F_{L}} \cdot \mathcal{I}_{4}^{\prime}$ and heterotic theory on $\left(T^{4} \times\left(T^{4}\right)^{\prime}\right) / \mathcal{I}_{20,4} \cdot \mathcal{I}_{4}^{\prime}$}

Type IIA theory compactified on $K 3$ is expected to be dual to the heterotic string theory on $T^{4}$. As was shown in ref.[2], under this duality, the transformation $(-1)^{F_{L}}$ in the type IIA theory gets mapped to $\mathcal{I}_{20,4}$ in the heterotic theory, where $\mathcal{I}_{20,4}$ denotes the change of sign of all the coordinates on the signature $(20,4)$ Narain lattice. Let us compactify the theory on a further four torus (which we shall denote by $\left.\left(T^{4}\right)^{\prime}\right)$ and mod out the theories by combined operation of $(-1)^{F_{L}}$ (or $\mathcal{I}_{20,4}$ ) and the reflection $\mathcal{I}_{4}^{\prime}$ on this new torus. This leads to the dual pair of theories described in the title of this subsection.

The spectrum in the twisted sector of the first theory can be analysed as in the previous example. By an $R \rightarrow(1 / R)$ duality transformation in one of the circles of $\left(T^{4}\right)^{\prime}$ this theory gets mapped to type IIB on $\left(K 3 \times\left(T^{4}\right)^{\prime}\right) / \mathcal{I}_{4}^{\prime}$, i.e. type IIB on $K 3 \times K 3^{\prime}$ in the orbifold limit of $K 3^{\prime}$. The twisted sector states come from the sixteen fixed points of $\left(T^{4}\right)^{\prime}$ under $\mathcal{I}_{4}^{\prime}$. In the original version of the theory before the $T$-duality transformation, these states could be interpreted as living on the sixteen NS five-branes of the type IIA theory transverse to $\left(T^{4}\right)^{\prime}$, and wrapped around $K 3$. In the six dimensional language, this represents sixteen dual strings moving on $\left(T^{4}\right)^{\prime} / \mathcal{I}_{4}^{\prime}$. Note that this theory does not have any tadpole of the $B_{\mu \nu}$ field[7], thus we do not get any extra elementary type II strings in this theory (unlike in the case of type IIA theory on $K 3 \times K 3$ ).

Let us now turn to the heterotic side of the story. The transformation $\mathcal{I}_{20,4} \times \mathcal{I}_{4}^{\prime}$ represents the change of sign of all the directions of the signature $(24,8)$ Narain lattice representing heterotic string compactification on $T^{8}$. Let us denote this transformation by $\mathcal{I}_{24,8}$. Modding out heterotic string theory on $T^{8}$ by $\mathcal{I}_{24,8}$ gives a consistent modular invariant theory, since 24 is a multiple of 8 and hence there is no problem with level matching between the left and the right sectors. Examining the twisted sector states, however, we discover that there are no massless states from the twisted sector. This is due to the fact that twenty four left-moving twisted bosons contribute a total vacuum energy of $1 / 2$. This seems to lead to an apparent contradiction, since the orbifold of the type IIA theory has massless states from the twisted sector. The resolution to this comes from the fact that the heterotic theory again has a one loop tadpole of $B_{\mu \nu}$ field of the form (2.1), which forces us to introduce $|n|$ elementary heterotic strings as background. In hindsight, this is precisely what we should have expected. On the type IIA side the twisted sector states live on sixteen dual strings, which under string-string duality transformation get 
mapped to sixteen elementary heterotic strings. Thus in order to show that the spectrum of massless states in these two theories coincide, all we need to show is that in this case $|n|$ equals 16 .

The computation of $n$ in the heterotic string theory proceeds in the same way as in the type IIA case and the final formula for $n$ is identical to the one given in eq.(2.3). There are two factors of 2 compared to the type II calculation which cancel. First of all GSO projection gives only a factor of $(1 / 2)$ in this case instead of a factor of $(1 / 4)$, since we have GSO projection only on the right. Also in this case spin structure arises only in the right sector, and unlike in the type II case, where (odd,even) and (even,odd) spin structures give equal contribution giving an extra factor of 2 , here the only contribution comes from the odd spin structure on the right without giving this extra factor.

Thus in order to calculate $n$, we need to calculate $A_{M}(q)$ for the conformal field theory describing the dynamics of transverse and internal coordinates of the heteroic string. This receives contribution from the Ramond sector in the right. As in the type IIA case we shall expand $A_{M}(q)$ in powers of $q$ and keep only terms upto order $q^{0}$. The twisted sector does not contribute, since the total vacuum energy of twenty four left-moving bosons exceeds zero. Untwisted sector contribution is given by:

$$
A_{M}(q)=-8\left(q^{-1}-24+O(q)\right)
$$

This arises in the following way. First of all the overall minus sign in $A_{M}(q)$ is due to the fact that the trace in this case is taken over the space-time fermionic states. There are sixteen Ramond sector ground states on the right, which transform in the $\left(8_{s}+8_{c}\right)$ representation of $\mathrm{SO}(8)$. Of these $8_{s}$ is odd and $8_{c}$ is even under $(-1)^{f_{R}}$ as before. Also $8_{s}$ is odd and $8_{c}$ is even under $\mathcal{I}_{24,8}$. The unique ground state on the left is even under $\mathcal{I}_{24,8}$ and hence must be combined with the state $8_{c}$ on the right to give an $\mathcal{I}_{24,8}$ invariant state. These eight states have vacuum energy of -1 from the left, and are even under $(-1)^{f_{R}}$; hence they give a contribution of $8 q^{-1}$. The first excited state on the left is twenty four fold degenerate and is odd under $\mathcal{I}_{24,8}$. Thus it must be combined with $8_{s}$ on the right to give an $\mathcal{I}_{24,8}$ invariant state. These $24 \times 8$ states have vanishing vacuum energy from the left sector, and are odd under $(-1)^{f_{R}}$; hence they contribute $-8 \cdot 24$.

Substituting (2.13) and (2.4) into (2.3) we get

$$
|n|=16
$$

as is required for getting duality invariant spectrum of massless states. 


\subsection{Type IIA on $\left(K 3 \times\left(T^{4}\right)^{\prime}\right) /(-1)^{F_{L}} \cdot \sigma_{I I} \cdot \mathcal{I}_{4}^{\prime}$ and heterotic theory on $\left(T^{4} \times\left(T^{4}\right)^{\prime}\right) / \mathcal{I}_{20,4} \cdot \sigma_{H} \cdot \mathcal{I}_{4}^{\prime}$}

We consider type IIA theory compactified on a special class of $K 3$ surfaces which have a $Z_{2}$ isometry generated by $\sigma_{I I}$ with the following properties [16, 17, 18]:

1. It exchanges the two $E_{8}$ factors in the lattice of second cohomology elements of $K 3$.

2. It has eight fixed points on $K 3$.

3. Modding out by this symmetry gives us back an orbifold of $\mathrm{SU}(2)$ holonomy.

The corresponding transformation $\sigma_{H}$ in the dual heterotic string theory on $T^{4}$ simply exchanges the two $E_{8}$ gauge groups in the theory. We now further compactify both theories on a four torus $\left(T^{4}\right)^{\prime}$ and mod out the type IIA theory by $(-1)^{F_{L}} \cdot \sigma_{I I} \cdot \mathcal{I}_{4}^{\prime}$ and the heterotic theory by its image $\mathcal{I}_{20,4} \cdot \sigma_{H} \cdot \mathcal{I}_{4}^{\prime}$. This leads us to the dual pair described above.

We shall now compare the spectrum of massless states in the twisted sector in the two theories. By making an $R \rightarrow(1 / R)$ duality transformation on one of the circles of $\left(T^{4}\right)^{\prime}$ we can map the type IIA theory to type IIB on $\left(K 3 \times\left(T^{4}\right)^{\prime}\right) / \sigma_{I I} \cdot \mathcal{I}_{4}^{\prime}$. In this theory there are $8 \times 16$ fixed points, since $\sigma_{I I}$ has eight fixed points on $K 3$ and $\mathcal{I}_{4}^{\prime}$ has sixteen fixed points on $\left(T^{4}\right)^{\prime}$. The twisted sector at each of these fixed points is characterized by eight anti-periodic bosons on the left and eight anti-periodic bosons on the right. The eight fermions on either side are periodic in the NS sector and anti-periodic in the R sector. As a result the total ground state energy in either side is zero in the R sector and one in the NS sector. Thus the only massless states in the twisted sector arise from the ground state of the RR sector. This state is unique since all the fermions are anti-periodic. Furthermore, this state, as constructed, is chiral, since in the light cone gauge that we are using the $k^{+}$component of the momentum is constrained to be non-vanishing. It is easy to verify by working in the covariant formulation that there are no massless states in this theory from the twisted sector with non-vanishing $k^{-}$. Thus each fixed point gives a massless chiral boson, giving a total of 128 massless chiral bosons in this theory. One can also verify that there are no $B_{\mu \nu}$ tadpoles in this theory since the type IIB theory is invariant under a world-sheet parity transformation under which $B_{\mu \nu}$ changes sign[0]. Thus we do not need to introduce any background elementary type II string. 
Let us now turn to the twisted sector states in the heterotic theory. There are altogether $16 \times 16$ fixed points since $\mathcal{I}_{20,4}$ has sixteen fixed points on $T^{4}$ and $\mathcal{I}_{4}^{\prime}$ has sixteen fixed points on $\left(T^{4}\right)^{\prime}$. The action of the $Z_{2}$ transformation on the sixteen internal left moving bosons is to exchange eight of them with eight others. Thus by taking appropriate linear combinations of these bosons we get eight periodic and eight anti-periodic internal bosons in the twisted sector. On the other hand all the eight bosons associated with the eight coordinates labelling $T^{4} \times\left(T^{4}\right)^{\prime}$ are anti-periodic both in the left and the right moving side. Thus we have a total of eight periodic and sixteen anti-periodic bosons on the left, giving a total vacuum energy of zero. On the right hand side we have eight anti-periodic bosons. In the NS sector, the eight fermions on the right are periodic, giving a total vacuum energy one, whereas in the $\mathrm{R}$ sector the eight fermions on the right are anti-periodic, giving a total vacuum energy zero. Thus we get a unique massless state from the Ramond ground state on the right. This is a fermionic state, and as before one can verify by working in the covariant formalism that these are chiral fermions. Thus we get a total of 256 chiral fermions from the 256 fixed points. Using Bose-Fermi equivalence in two dimensions these can be shown to be equivalent to 128 chiral bosons. Thus we again get identical spectrum of massless fields from the twisted sector of the two theories.

It remains to verify that there is no $B_{\mu \nu}$ tadpole in this theory, since any such tadpole will force us to introduce heterotic string background and hence introduce new massless states from the collective coordinates of these strings. The calculation proceeds as in the last subsection. In particular, the untwisted sector contribution to $A_{M}(q)$ is now given by

$$
-8\left(q^{-1}-8+O(q)\right) .
$$

Note that the 24 in eq.(2.13) has been replaced by 8 , since of the 24 left moving bosonic oscillators 16 are odd and 8 are even under $\mathcal{I}_{20,4} \cdot \sigma_{H} \cdot \mathcal{I}_{4}$. Thus 16 of these oscillator states need to be accompanied by $(-1)^{f_{R}}=-1$ states from the right, and 8 of them have to be accompanied by $(-1)^{f_{R}}=1$ states from the right. This time there is also twisted sector contribution to $A_{M}(q)$. The 256 massless twisted sector states, each with $(-1)^{f_{R}}=1$, contributes a factor of -256 to $A_{M}(q)$. (The -1 is again due to the fact that these states are all space-time fermions.) This gives

$$
A_{M}(q)=-8\left(q^{-1}+24+O(q)\right) .
$$

Substituting this and (2.4) into (2.3) we get

$$
n=0 \text {, }
$$


as desired.

\section{Conclusion}

In this paper we have constructed several examples where the orbifolding procedure commutes with the duality transformation. We start with a known dual pair of theories, identify a pair of symmetries in these two theories that are related by a duality transformation, and mod out both theories by their respective symmetries to construct a dual pair. In most cases, if we do not combine the original pair of symmetries with a space-time symmetry transformation, we are lead to an inconsistent result. On the other hand, if we combine the original pair of symmetries (which could be called internal symmetries) with a space-time symmetry transformation (with fixed points in general), and then construct the orbifold, we get a consistent dual pair. In many of these cases, we get identical spectrum of massless states in the dual pair of theories constructed this way only after introducing appropriate background fields that cancel the one loop tadpoles in both the theories. This puts non-trivial constraint on the coefficients of one loop tadpoles in various theories, and in every case that has been studied one finds that the coefficient of the tadpole is consistent with the predictions of duality.

Many examples of dual pairs of theories, constructed by modding out another dual pair by appropriate symmetries, have been discussed before 11, 2, 9, 19. Our examples differ from most of the previous examples in that in our models, there are massless states from the 'twisted sector' in both theories at a generic point in the moduli space. So far there has been no systematic rule for determining when orbifolding commutes with duality transformation, except in cases where the adibatic argument of ref. [2] is applicable. We hope that the results of this paper will provide a step towards a more systematic understanding of this phenomenon.

Finally the result of this paper boosts our confidence in the results of ref. [3] where many of the conjectures involving orbifolds of $M$-theory were derived using the ansatz that orbifolding procedure commutes with the duality transformation. The only place where this procedure failed was in finding the dual of the $Z_{2}$ orbifold of $M$ theory on $S^{1}$, where it gave the dual theory as type IIB string theory in ten dimensions instead of the $E_{8} \times E_{8}$ heterotic string 20]. According to the classification given in this paper, this example falls in the class 3 , where the argument for duality is the weakest, and fails even 
in many string theory examples. On the other hand, every other example discussed in ref. [3], where this procedure gave sensible answer, is of type 2(b) in our classification. As we saw in this paper, in many string theory examples of this kind we get sensible answers by assuming that orbifolding commutes with the duality group. It is satisfying that even in the $M$-theory examples we got sensible answers precisely for this class of models.

I wish to thank K. Dasgupta and S. Mukhi for useful discussions. I would also like to thank the theoretical high energy physics group at Rutgers university for hospitality during the course of this work.

\section{References}

[1] S. Ferrara, J. Harvey, A. Strominger and C. Vafa, Phys. Lett. B361 (1995) 59 hepth/9505162].

[2] C. Vafa and E. Witten, hep-th/9507050.

[3] A. Sen, hep-th/9603113.

[4] B. Acharya, hep-th/9603033.

[5] K. Dasgupta and S. Mukhi, hep-th/9512196.

[6] E. Witten, hep-th/9512219.

[7] C. Vafa and E. Witten, Nucl. Phys. B447 (1995) 261 hep-th/9505053].

[8] C. Hull and P. Townsend, Nucl. Phys. B438 (1995) 109 hep-th/9410167.

[9] A. Sen and C. Vafa, Nucl. Phys. B455 (1995) 165 hep-th/9508064.

[10] E. Gimon and J. Polchinski, hep-th/9601038;

J. Polchinski, S. Choudhuri and C. Johnson, hep-th/9602052;

and references therein.

[11] E. Witten, Nucl. Phys. B443 (1995) 85 hep-th/9503124.

[12] D. Kutasov, hep-th/9512145.

[13] M. Bershadsky, V. Sadov and C. Vafa, hep-th/9510225. 
[14] H. Ooguri and C. Vafa, hep-th/9511164.

[15] D. Kutasov, E. Martinec and M. O'Loughlin, hep-th/9603116.

[16] V. Nikulin, Trans. Moscow Math. Soc. 2 (1980) 71.

[17] J. Schwarz and A. Sen, Phys. Lett. B357 (1995) 323 [hep-th/9507027.

[18] S. Chaudhuri and D. Lowe, hep-th/9508144.

[19] A. Kumar, hep-th/9601067.

[20] P. Horava and E. Witten, Nucl. Phys. B460 (1996) 506 hep-th/9510209; hepth/9603142. 